\title{
A Modern View on the Surgical Treatment of Proximal Humerus Fractures (Literature Review)
}

\author{
Alimov AP ${ }^{1 *}$, Yusupov S Yu ${ }^{2}$ and Hakimov Sh $\mathrm{K}^{3}$ \\ ${ }^{1}$ Republican Specialized Scientific and Practical Medical Center of Traumatology and Orthopedics Ministry of Health of the \\ Republic of Uzbekistan,Uzbekistan
}

${ }^{2}$ Bukhara State Medical Institute, Uzbekistan

${ }^{3}$ Bukhara branch of the Republican Scientific Center for Emergency Medical Aid, Uzbekistan

*Corresponding author: Alimov AP, Republican Specialized Scientific and Practical Medical Center of Traumatology and Orthopedics

Ministry of Health of the Republic of Uzbekistan, Tashkent city, Uzbekistan

\section{ARTICLE INFO}

Received: May 13, 2020

Published: 幽 May 21, 2020

Citation: Alimov AP, Yusupov S Yu, Hakimov Sh K. A Modern View on the Surgical Treatment of Proximal Humerus Fractures (Literature Review). Biomed J Sci \& Tech Res 27(5)-2020. BJSTR. MS.ID.004563.

\section{Abstract}

This scientific work is devoted to a review of literary sources regarding the surgical treatment of patients with fractures of the proximal humerus, which showed divergence of opinions between specialists in regards to the choice of optimal strategy for surgical treatment of patients of different age groups. Despite the development of a wide range of surgical methods and methods of fixation, various complications are observed in the postoperative period, which indicates the need for further optimization of methods for surgical correction of fractures of this localization.

Keywords: Humerus; Proximal Humerus Fractures; Osteosynthesis; Plate

\section{Relevance of the Subject}

According to the WHO, fractures of the surgical neck of the humerus account for approximately $5-15 \%$ of all injuries of the human skeleton bones, moreover in $60 \%$ of cases they are characterized by multi-fragmented fractures and are accompanied by a pronounced displacement of the fragments, which contribute to special difficulties in choosing the tactics of surgical treatment or reposition of bone fragments with conservative treatment [1]. The severity of displacement of bone fragments depends on the energy strength of the injury, which in $85 \%$ of cases is characterized by the absence of displacement of bone fragments due to low-energy injuries, in which surgical intervention is not required, and they are treated conservatively. However, a correct assessment when developing indications for conservative treatment allows achieving good functional results in $80-87 \%$ of cases [2,3]. Difficulties in reposition of bone fragments according to AA Kolomiyets et al. (2006) up to $50 \%$ of cases are caused by interposition of soft tissues between fragments that prevent closed reposition $[4,5]$.

Despite the high achievement in the surgical treatment of fractures of the surgical neck of the humerus according to
E. Weber et al. (1998) complete restoration of damaged limb function was noted only in $38 \%$ of cases $[5,6]$. Most authors believe that conservative treatment should not be abandoned, while others consider it to be ineffective. In general, the result of treatment depends on the severity of the injury, the type of fracture, the quality of the reposition, and also to a large extent on the patient's persistence and level of motivation. The best results are observed in active patients with a high level of self-discipline $[3,7]$. The domestic literature describes more than 30 different methods of surgical treatment of fractures of the surgical neck of the humerus. Most often authors use: osteosynthesis of external fixation devices of various design, fixation of bone fragments with transossal sutures, osteosynthesis with pins, plates and screws , intramedullary osteosynthesis (with blocking and using rigid constructions of the "TEN-pin" type), as well as the establishment of a unipolar endoprosthesis of the shoulder joint in cases of severe destruction of the proximal humerus $[7,8]$.

Handoll H.H. et al (2015) divided the surgical methods of treatment for fractures of the proximal humerus into the following: 
1. Closed reduction and percutaneous fixation with pins;

2. Extrafocal fixation;

3. Open reposition and internal fixation by a plate;

4. Open reposition and fixation by tightening loop method;

5. Intramedullary fixation;

6. Hemiartroplasty (prosthesis of the humeral head);

7. A total shoulder joint prosthesis (anatomical or reverse) $[4,9]$.

Despite the development and widespread use of quite a few different methods of surgical intervention, postoperative complications and unsatisfactory results range from 12 to $35 \%$ in the form of instability of the installed plate, nail migration, the appearance of impingement syndrome, aseptic necrosis of the humeral head $[10,11]$. When choosing the operational method for stabilization of fragments, it is necessary to focus on the data of the X-rays taking into account the state of the bone tissue, the size and number of bone fragments in which the choice of tactics in each case is individual $[12,13]$. As known, good bone consolidation of fractures of the surgical neck of the humerus in patients can be achieved only after adequate reduction of bone fragments in the open. The group of authors believes that it is necessary to operate patients at the earliest possible time and surgery technique should be minimally invasive, using low-traumatic fixatives to prevent the development of complications such as avascular necrosis. The very beginning of the history of the surgical treatment of patients with fractures of the surgical neck of the humerus is associated with the use of pins as an osteosynthesis.

Closed percutaneous pinning can be performed in patients with biphasic proximal shoulder fractures with good bone density with the appropriate equipment - an electron-optical transducer $[14,15]$. Due to insufficient stability of osteosynthesis with pins and for the prevention of pin migration S.I. Makarova et al. (2007) after closed reposition and fixation with pins, gypsum limb immobilization is applied for 3-4 weeks. Other authors adhere to similar tactics when performing gypsum immobilization after osteosynthesis with pins for a period of two to six weeks $[16,17]$. All known methods of percutaneous fixation with pins have common drawbacks: difficulty achieving anatomical reposition, insufficient stability of osteosynthesis, risk of damage to blood vessels and nerves during pinning (Pankov I. O., 2003). Nevertheless, at present, many foreign authors consider this technique to be a method of choice for multifragment fractures, especially in elderly patients . Jacob in 1991 revealed the development of avascular necrosis in $26 \%$ of cases with percutaneous fixation, and in $2008 \mathrm{R}$. Bogner et al. obtained a significantly better result in $3 \%$ of cases $[12,18]$.

The rapid development of the history of surgical treatment of fractures of the proximal end of the humerus began with the use of

Copyright@ Alimov AP | Biomed J Sci \& Tech Res | BJSTR. MS.ID.004563. an external fixation apparatus by the American surgeon J. Emsberry in 1831 [19]. In 1951 G.A. Ilizarov proposed an apparatus for transosseous osteosynthesis (TOO), which differs from others in its expanded ability to move bone fragments and low invasiveness, the prototype of which is the Witmozer's device. According to N.V. Tyulyaev et al. (2011) external fixation devices are used for diaphyseal fractures of the upper limbs in $25-42 \%$ of cases with closed injuries and in $50 \%$ with open $[11,20]$. Based on data from 514 patients with fractures of different levels of the humerus Raengulov (2000) gives recommendations on the choice of surgical treatment. External fixation devices are advisable in order to reduce the morbidity and complexity with closed unstable fractures of the humerus, open and multi-fragmented, contaminated fractures, and only in urgent order, creating conditions for temporary fixation of fragments and for the implementation of subsequent measures [5,21].

A common drawback of external fixation devices is that they do not always allow the efficient reposition of fragments, especially in cases of chronic fractures, comminuted fractures, in the presence of angular and rotational displacements. Furthermore, the imposition of external fixation devices is a rather complicated surgical operation and in some cases presents serious inconvenience Makarova S. I., 2007. An individual differentiated approach to the tactics of surgical treatment for fractures-dislocations of the proximal humerus in adults interested many scientists, which led to the development of various fixation devices, from pin fixation to more modern, biological types of osseous fixators [7,22]. E. Sh. Lomtatidze et al. (2003) while analyzing the functional results of surgical treatment of proximal humerus fractures while considering the age and nature of the fracture, concluded that osteosynthesis with plates and nails is more appropriate for young patients to obtain the best long-term results, and in elderly patients over 60 years of age the most delicate and at the same time reliable fixation method is recommended [15,23].

In case of multi-fragmented (three- and four-fragmented) fractures, caused by high-energy injuries, it is impossible to achieve sufficient adequate fixation by using pin fixation, which led to the development and use of more optimal types of plates, such as BIOS, LCP, DCP, providing stable fixation of fragments that do not violate the normal anatomy and functionality of the shoulder segment $[24,25]$. Since L. Böhler declared in 1964 that the treatment of fractures of the humerus should be exclusively surgical, a lot of time has passed and now surgical treatment is the "gold standard" due to the development of modern methods of intramedullary and bone osteosynthesis. The first application of intramedullary osteosynthesis of the humerus belongs to Kunchery. In the first half of the twentieth century, fractures of the humerus due to the large number of complications associated with the migration of the structure, and the lack of rotational stability made us look for new ways to solve the existing problem. Indications for intramedullary osteosynthesis for fractures of the proximal humerus are limited. 
It is used in patients with two-fragment fractures. With this method of treatment, the risk of damage to the radial and axillary nerves by nails is high (Kies T.R., 2001). Improving the technique of open intramedullary osteosynthesis in 1974, A. Kapandij proposed intramedullary rods for the treatment of stable subcapital fractures [26]. In 1984 H. Seidel proposed HLN (humeral locked nail) - a pin for the humerus, having a locking hole at the apex, which allows stable fixation of fragments of fractures of the head of the humerus [27]. The advantages of the open reposition and internal fixation method (ORIF) are anatomical reconstruction, early mobilization of the limb and a good functional result. However, there is an opinion that "even technically competently performed fixation with a highquality implant does not always allow to avoid the subsequent migration of nails or a fracture of the plate, and consolidation in the correct position is not guaranteed in the future" [28]. With fractures and dislocations of the proximal humerus, indicators of avascular necrosis development vary within $35 \%$ with ORIF methods [17] compared with closed reposition and fixation of fragments by pins, which in the latter occurs in $2.4 \%$ to $11 \%$ cases $[5,20]$.

In one of his work, P. Gierer (........) explains the reason for the development of avascular necrosis with osteosynthesis of the humeral head in cases where the fracture line passes through the articular surface [18]. The application of the above mentioned methods is targeted only for stable options of fractures of the proximal end of the humerus, which prompted scientists to develop optimal osteosynthesis methods that provide stable fixation simultaneously with stable, as well as unstable fractures with multi-fragmented humerus fractures, especially in elderly patients $[18,19]$. Given the global trend to perform minimally invasive and less traumatic surgeries, as well as the need to restore the function of the operated limb as soon as possible, closed intramedullary osteosynthesis with proximal blocked shafts can be considered one of the most promising methods of treatment for comminuted fractures of the proximal humerus [4,5]. In recent decades, a lot of scientific works have appeared in the literature dedicated to closed reposition with blocked intramedullary osteosynthesis (BIOC) of the humerus for fractures of the proximal humerus representing a number of advantages, such as the absence of an open reposition of bone fragments with preservation of biological material (primary hematoma) for complete osteoreparation , the possibility of stable fixation of the fracture area due to the installation of multi-plane nails on the proximal and distal parts of the segment and early development of a segment, providing stimulation of bone fusion and prevents contracture and stiffness in adjacent joints.

The BIOS technique has become widely used among many large schools and has led to the development of more standardized device options, after analyzing its results and identifying shortcomings $[14,18]$. The earliest versions of the intramedullary pins, such as the Rush shaft, did not provide adequate fixation stability, including rotational, which led to the migration of retainers and required repeated surgical interventions. The weak point of the second generation lockable pins such as Polarus nail and Targon PH is weak fixation with proximal locking nails, which can lead to the migration of pins. [28,29]. Continuing the development of advanced third generation options, such as the Stryker T2 Proximal Humeral Nail and Synthes Proximal Humeral Nail, showed sufficient fixation strength and stability of proximal locking nails. The solution to the problem of pin migration is based on the implementation of nailto-nail, spiral blade technology. The use of a proximal shoulder pin allows minimally invasive osteosynthesis with the lowest risk of infectious complications compared to other surgeries.

However, osteosynthesis with a standard proximal shoulder pin can damage the tendons of the rotator cuff and cause postoperative pain in the shoulder joint. A proximal lockable plate is better for fixation of fractures in young patients, but the fixation of fractures in patients with osteoporosis remains a problem. In $40 \%$ of cases, complications when using plates are associated with errors in surgical technique. Plate fractures occur in $1.9 \%$, impingement in $2.6 \%$, the formation of a false joint - in $2.6 \%$, wound infection - in 3.9\%, loss of reposition - in 7.1\%, necrosis of the humeral head - in 3.9\% of cases [21].Scientists at the Clinic of the Republican Specialized Scientific and Practical Medical Center for Traumatology and Orthopedics of the Republic of Uzbekistan offer a pin-shaft apparatus developed in the clinic for three- and fourfragment fractures of the proximal humerus in elderly patients. The installation of a pin-shaft apparatus is very easy and less traumatic when used with reliable fixation and preservation of motor function in the shoulder joint, a patent for a utility model was obtained (FAP 20100015).

The history of bone osteosynthesis dates back to 1969, when the AO group proposed the use of T-shaped plates together with large spongy nails. Until the early 2000s, they were used exclusively for AO type A2 fractures, and for B and C type fractures this technique was recognized as ineffective since it was often accompanied by complications, such as the instability of fragments due to the instability of the metal structure itself, impingement of a massive structure with an acromial process of the scapula, often leading to revision intervention [28]. Currently, the use of various types of plates, such as plates of CITO, AO / ASIF, LSP, DSP, etc., are referred to as bone osteosynthesis. One of the founders of AO (Association for the Study of Internal Fixation Methods of AO / ASIF) is Robert Danis (1880 - 1962), who believes that based on functional reasons, each fracture in itself is already an indication for surgical treatment. In his opinion, surgery should be performed as soon as possible.

AO / ASIF itself was created in 1958 and four basic principles of osteosynthesis were then formulated:

1. Anatomical reposition (perfect matching of fragments during surgery)

2. Rigid stable fixation. 
3. Preservation of blood supply to bone fragments.

4. Early active movements.

Also today, many authors support the above principles in their functional activities.

At one time, AS / ASIF plates were rational in use, became widespread in all developed schools of the world, led to many controversial disagreements with large-scale use, on the basis of which, new improved bone retainers were developed [22,27]. The only drawback of the AO / ASIF plate is the lack of compression when it is installed on the surface of the cortical layer of the bone due to classic simple holes, and also is not a retainer of choice in patients with multi-fragmented fractures arising from osteoporosis $[5,30]$. The unresolved issue is the choice of the type of fixation between the plate and the shaft for fractures of the proximal humeral metaepiphysis. According to many researchers, when comparing the type of osteosynthesis with three- and four-fragment fractures of the proximal humerus, there is a significant difference between the use of two types: lockable plates and lockable shafts from a position of stability is not detected, however, osteosynthesis with a shaft can be performed less invasively. Many scientists explain that the expression "stability" can be either absolute or relative, reaching during surgery depending on the location of the fracture.

For diaphyseal fractures (femoral, humerus fracture in the middle third) it is enough to: restore the segment length, get rid of angular deformation and rotational displacement. Therefore, in place of absolute stability in the treatment of diaphyseal fractures - stability is quite relative. A consequence of achieving relative stability is fusion with the formation of periosteal callus [1]. On this basis, already in the late 80 s - early 90s, coming from the surgical principles of $\mathrm{AO}$, the first - "anatomical reposition" was rephrased to "functional reposition". The plates are used in two directions, with the aim of neutral fixation in comminuted and multifragmented fractures and to create conditions for compression or dynamization in the absence of fragments. Deriving from that plates are distinguished as follows: 1) plates with round holes; 2) plates with oval holes; 3) dynamically compressing plates; 4) plates with angular stability of the nail. Currently, plates with dynamic compression are the most commonly used: DCP (S. Perren et al 1969) and LC-DCP (S. Perren et al. 1989).

The configuration of the holes of the plates with dynamic compression is that at the final stage of introducing the nail into the bone, its head "slides" towards the middle of the plate, whereby the convergence of fragments occurs over the area of the fracture fragments. The possibilities of bone osteosynthesis expanded significantly with the advent of plates with angular stability of nails, since they allow stable fixation with relatively small dimensions of the structure [14]. In the presence of osteoporosis, osteopenia in patients with three-, four-fragment fractures, osteosynthesis with proximal shoulder plates with angular stability is indicated
(LCP). The advantages of using such plates are obvious. These include stable fixation of the fracture, a decrease in the likelihood of secondary displacement of fragments due to fragment lysis. This allows you to start earlier movement development[16]. Data from PubMed.gov. being one of the largest information resources, showed the results of the technique, universally recognized as the "gold standard" - osteosynthesis with LCP-type plates in 340 patients over the period from 1992 to 2012.

Both methods have a large number of supporters and opponents, since both have disadvantages and certain advantages as well $[12,13]$. According to A.M. Foruria lacks rotational stability in the broken segment when using LCP plates as osteosynthesis, led to the development of LPHP plates, which have greater resistance to torsion loads The angular stability and rotational stability of the LPHP type plate are due to the location of the installed nails under different angular flatnesses relative to each other, which ensures rigid fixation of the shoulder head to the plate, and also does not exert pressure on the periosteum and cortical layer of the bone itself, thereby not disturbing blood circulation in the bone fragments [2,31]. Plate osteosynthesis requires extensive access, which, in turn, increases the risk of osteonecrosis of the humeral head due to impaired vascularization. Furthermore, rather bulky bone constructions can cause subacromial impingement, and in the presence of osteoporosis there is a risk of failure of the bone fixation. The disadvantage of plate osteosynthesis without angular stability of the nails is also the development of plate instability, including accompanied by its fracture [28].

For several years, many authors proposed in the case of three- and four-fragment fractures to do primary hemiartroplasty (according to Neer) or to perform unipolar shoulder joint replacement taking into account the inefficiency and many disadvantages of the known bone and intraosseous methods of osteosynthesis [32]. Despite the large number of conservative and surgical methods used to treat patients with injuries of the proximal humerus, there is no unified approach to the choice of a particular treatment option depending on the age of the patient, the nature of the displacement of bone fragments and the duration of the injury. Given the diverse nature of fractures of the proximal humerus, the choice of treatment for patients with fractures of the proximal humerus should be individual. Taking into account the above data on the analysis of numerous literature sources, it should be noted that the issues of surgical correction of fractures of the proximal end of the humerus remain one of the unresolved chapters of modern traumatology. Despite the developments, the majority of operational methods and modern fixation devices with biological significance, the percentage of unsatisfactory results and various problems of difficult to solve complications remains quite high, which on the whole represents the real relevance of this work and needs to be considered as a promising area for further research work. 


\section{References}

1. Sun JC, Li YL, Ning GZ, Wu Q, Feng SQ, et al. (2013) Treatment of threeand four-part proximal humeral fractures with locking proximal humerus plate. Eur J Orthop Surg Traumatol 23(6): 699-704

2. Keener JD, Parsons BO, Flatow EL, Rogers K, Williams GR, et al. (2007) Outcomes after percutaneous reduction and fixation of proximal humeral fractures. J. Shoulder Elbow Surg 16(3): 330-338.

3. Loskutov AE (2007) Differencirovannye well-being to OperativnomuLecheniyu was 'nyh with Povrezhdeniyamiproksimal' NogoOtdelaplechevoi Bone. Orthopaedics, traumatology and prosthetics 1:33-38.

4. CM (2011) Modern perspectives of open reduction and plate fixation of proximal humerus fractures. J Orthop Trauma 25(10): 618-629.

5. Robinson BC, Athwal GS, Sanchez Sotelo J, Rispoli DM (2008) Classification and imaging of proximal humerus fractures. Orthop Clin North Am 39(4): 393-403.

6. Brunner A (2012) Minimally invasive percutaneous plating of proximal humeral shaft fractures with the Proximal Humerus Internal Locking System (PHILOS). J Shoulder Elbow Surg 21(8): 1056-1063.

7. Slobodskoy AB (2011) Morphological changes in the head of the humerus in acute injury of the proximal humerus in elderly patients. In: Ilizarov readings: materials of the scientific and practical conference with international participation. Kurgan 260-261.

8. Wong J, Newman JM, Gruson KI (2016) Outcomes of intramedullary nailing for acute proximal humerus fractures: a systematic review. J Orthop Traumatol 17: 113-122.

9. Dilisio MF, Nowinski RJ, Hatzidakis AM (2016) Intramedullary nailing of the proximal humerus: evolution, technique, and results. J Shoulder Elbow Surg 25(5): e130-e138.

10. Zhu Y, Lu Y, Shen J, Zhang J, Jiang C, et al. (2011) Locking intramedullary nails and locking plates in the treatment of two-part proximal humeral surgical neck fractures: a prospective randomized trial with a minimum of three years of follow-up. J Bone Joint Surg Am 93(2): 159-168.

11. Martin C, Guillen M, Lopez G (2006) Treatment of 2- and 3-part fractures of the proximal humerus using external fixation: a retrospective evaluation of 62 patients. Acta Orthop 77(2): 275-278.

12. Magovern B (2008) Percutaneous fixation of proximal humerus fractures. Orthop Clin North Am 39: 405-416.

13. Minayev AN, Gorodnichenko AI, Uskov ON (2010) The transosseousosteosynthesis by proximal metaepiphyseal fractures of humerus in elderly patients. Khirurgiya (1): 50-53.

14. Gavaskar AS, Karthik BB, Tummala NC, Srinivasan P, Gopalan H, et al. (2016) Second generation locked plating for complex proximal humerus fractures in very elderly patients. Injury 47(11): 2534-2538.

15. Iyengar JJ, Devcic Z, Sproul RC, Feeley BT (2011) Nonoperative treatment of proximal humerus fractures: a systematic review. J Orthop Trauma 25(10): 612-617.

16. Yang KH (2005) Helical plate fixation for treatment of comminuted fractures of the proximal and middle one-third of the humerus. Injury 36 : 75-80.
17. Gurkin BE (2015) Basis for choice of treatment of patients with fracture of the proximal humeral. Kubanskiynauchnyymeditsinskiyvestnik (2): 49-56.

18. Minayev AN, Gorodnichenko AI, Uskov ON (2010) Thetransosseousosteosynthesis by proximal metaepiphyseal fractures of humerus in elderly patients. Khirurgiya(1): 50-53.

19. Bengner $U$ (1998) Changes in the incidence of fracture of the upper end of the humerus during a 30-year period. ClinOrthop 231: 179-182.

20. Duralde XA, Lee R (2010) The results of ORIF of displaced unstable proximal humeral fractures using a locking plate. J Shoulder Elbow Surg 19(4): 480-488

21. Eldzarov PE, Zelianin AS, Filippov VV (2010) Surgical treatment of consequences of femoral fractures. Khirurgiya (9): 47-52.

22. Skoroglyadov AV (2008) Possibility of Ultrasound Diagnosis for Visualization of Peripheral Nerves of and Forearm. Bulletin of Traumatology and Orthopedics named after NN Priorov (2): 64-69.

23. Mora Guix JM, Juan SalaPedrós, Alejandro Castaño Serrano (2009) Classification System for Proximal Humeral Fractures. Clin Med Res 7(1-2): 32-44.

24. Kogan P (2013) Treatment evolution of proximal humeral fractures (literature review)]. [Traumatology and Orthopaedics of Russia (3): 154161.

25. Buecking B, Juliane Mohr, Benjamin Bockmann, Ralph Zettl, Steffen Ruchholt, et al. (2014) Deltoid-split or Deltopectoral Approaches for the Displaced proximal humeral fractures? Clin Orthop Relat Res 472(5): 1576-1585.

26. Boudard G (2014) Locking plate fixation versus antegrade nailing of 3and 4- part proximal humerus fractures in patients without osteoporosis. Comparative retrospective study of 63 cases. OrthopTraumatolSurg Res 100(8): 917-924.

27. Lekic N, Nicole M Montero, Richelle C Takemoto, Roy I Davidovitch, Kenneth A Egol, et al. (2012) Treatment of two-part proximal humerus fractures: Intramedullary nail compared to locked plating. HSS J 8(2): 86-91.

28. Mora Guix JM (2009) Updated Classification System for Proximal $\mathrm{Hu}-$ meral Fractures. Clin Med Res 7(1-2): 32-44.

29. Den Hartog D, de Haan J, Schep NW, Tuinebreijer WE (2010) Primary shoulder arthroplasty versus conservative treatment for comminutedproximal humeral fractures: a systematic literature review. Open Orthop J 4: 87-92.

30. Murylev V, Imamkuliev A, elizarov P, Korshev O, Kutuzov A, et al. (2014) Surgical treatment of extra articular fractures of the proximal humerus. Vrach [Physician] (11): 10-13.

31. Rothstock S, Plecko M, Kloub M, Schiuma D, Windolf M, et al. (2012) Biomechanical evaluation of two intramedullary nailing techniques with different locking options in a three-part fracture proximal humerus model. Clin. Biomech. Bristol, Avon 27(7): 686-691.

32. Pritchett JW (1997) Inferior subluxation of the humeral head after trauma or surgery. J Shoulder Elbow Surg 6(4): 356-359. 
ISSN: 2574-1241

DOI: $10.26717 /$ BJSTR.2020.27.004563

Alimov AP. Biomed J Sci \& Tech Res

(C) (i) This work is licensed under Creative BY Commons Attribution 4.0 License

Submission Link: https://biomedres.us/submit-manuscript.php

$\begin{array}{ll}\text { BIOMEDICAL } & \text { Assets of Publishing with us } \\ \text { RESEARCHES } & \text { - Global archiving of articles } \\ \text { - Immediate, unrestricted online access } & \text { - Rigorous Peer Review Process } \\ & \text { - Authors Retain Copyrights } \\ & \end{array}$

